\title{
\begin{tabular}{l|l} 
POLITIQUES \& & Politiques et management public
\end{tabular}
}

\section{Le plafond de verre dans les ministères : regards croisés de la sociologie du travail et de la science politique}

Glass Ceiling in the Public Administration: Perspectives from Sociology of Work

and Political Science

Laure Bereni, Catherine Marry, Sophie Pochic et Anne Revillard

\section{(2) OpenEdition}

\section{Journals}

Édition électronique

URL : http://journals.openedition.org/pmp/4141

ISSN : 2119-4831

Éditeur

Institut de Management Public (IDPM)

Édition imprimée

Date de publication : 15 juin 2011

ISSN : 0758-1726

\section{Référence électronique}

Laure Bereni, Catherine Marry, Sophie Pochic et Anne Revillard, «Le plafond de verre dans les ministères : regards croisés de la sociologie du travail et de la science politique », Politiques et management public [En ligne], Vol 28/2 | 2011, mis en ligne le 03 octobre 2012, consulté le 21 décembre 2020. URL : http://journals.openedition.org/pmp/4141 


\title{
Le plafond de verre dans les ministères: regards croisés de la sociologie du travail et de la science politique
}

\author{
Laure Bereni ${ }^{a}$, Catherine Marry ${ }^{a *}$, \\ Sophie Pochic ${ }^{a}$, Anne Revillard ${ }^{b}$ \\ ${ }^{a}$ Centre Maurice Halbwachs - Equipe PRO \\ ${ }^{b}$ CERAL - UFR Droit, sciences politiques et sociales, Université Paris Nord
}

\section{Résumé}

Si les mécanismes du « plafond de verre » (barrière invisible qui empêche les femmes d'accéder aux hautes sphères du pouvoir, des honneurs et des rémunérations) ont fait l'objet de nombreuses recherches au cours des dernières années, peu de travaux ont exploré, de façon approfondie et systématique, les dynamiques de genre au sein de «l'aristocratie des fonctionnaires ». En croisant les apports des recherches en sociologie du travail et en science politique qui adoptent une perspective de genre, cet article propose un cadre analytique original pour comprendre la « fabrication » différentielle des carrières des hommes et des femmes dans la haute fonction publique.

(c) 2011 IDMP/Lavoisier SAS. Tous droits réservés

Mots clés : plafond de verre ; égalité professionnelle ; parité ; genre et organisations ; femmes cadres.

\section{Abstract}

Glass Ceiling in the Public Administration: Perspectives from Sociology of Work and Political Science. While a great deal of research has been devoted over recent years to "glass ceiling" mechanisms (invisible barrier that prevents women and minorities from rising to top positions of power, honors and earnings), there is a lack of in-depth studies on gender dynamics in the "civil servants aristocracy". Drawing on both sociological and political science research on gender, organizations and power, this article provides an original framework to understand men's and women's careers are differentially built in high civil service.

(c) 2011 IDMP/Lavoisier SAS. Tous droits réservés

Keywords: glass ceiling; workplace equality; gender parity; gender and organizations; women executives/women in leadership.

\footnotetext{
*Auteur correspondant : catherine.marry@ens.fr

doi:10.3166/pmp.28.139-155 @ 2011 IDMP/Lavoisier SAS. Tous droits réservés
} 


\section{Introduction}

L'essor des scolarités féminines et la féminisation des professions supérieures n'ont pas supprimé tous les obstacles auxquels se heurtent les femmes pour atteindre les hautes sphères du pouvoir, des honneurs et des rémunérations. La fonction publique n'échappe pas à ce phénomène. Le récent rapport Guégot (2011) ne recense ainsi que $20 \%$ de femmes dans les personnels de direction de la fonction publique d'Etat en 2008, alors qu'elles représentent $57 \%$ des cadres de catégorie A. Il souligne aussi les fortes variations selon les professions, qui traduisent la ségrégation horizontale ou « cages de verre » présente sur ce marché du travail du public comme dans le privé (cf. dans ce numéro la contribution de F. Milewski).

Les chantiers de recherche se sont multipliés ces dernières années en France comme ailleurs pour décrire et interpréter la disparition des femmes dans les hautes sphères. Ils revisitent la métaphore statique du « plafond de verre », à propos de professions très diverses, pour rendre compte de son historicité, souligner à la fois la dynamique générationnelle d'égalité mais aussi des formes de stagnation (Laufer, 2004 ; Buscatto et Marry, 2009; Marry et Le Mancq, 2011). Peu de travaux ont toutefois exploré, de façon approfondie et systématique, les dynamiques de genre au sein de « l'aristocratie des fonctionnaires », soit parmi les titulaires des postes de pouvoir dans les directions des ministères et de leur administration centrale. Ce numéro est une première étape dans ce sens. Le propos de cet article est de brosser un état des lieux de la littérature en sociologie du travail et en science politique adoptant une perspective de genre pour en tirer des fils ou hypothèses d'interprétation de la « fabrication » différentielle des carrières des hommes et des femmes dans la haute fonction publique ${ }^{1}$.

La perspective sociohistorique s'avère selon nous indispensable pour rappeler la progressive et sélective levée des barrières à l'entrée (formations ou concours) qui a permis aux femmes d'investir les premiers échelons de l'administration. Une fois entrées, ces femmes sont longtemps restées cantonnées aux échelons inférieurs (assistantes, collaboratrices, adjointes), secondaires (fonctions support ou expertes), voire périphériques des univers administratifs (auxiliaires, bénévoles). Des travaux sociologiques ont montré l'intérêt heuristique de regarder au niveau « micro » les ressources et contraintes des pionnières accédant aux postes de pouvoir traditionnellement masculins, à l'articulation entre vie personnelle et vie professionnelle. Ces biographies doivent être réencastrées dans des organisations publiques en constante transformation (critères et modalités de recrutement, filières de promotion, croissance ou réduction des effectifs) afin de décrypter au niveau « méso » la « structure des opportunités » de promotion qui s'offrent aux femmes diplômées (Kanter, 1977).

Les débats sur les réformes de la fonction publique en France abordent peu leurs effets induits sur les parcours des femmes, qui pourtant composent $60 \%$ de ses agents. Ces perspectives sociologiques peuvent être utilement complétées par des éclairages de science politique, à partir d'une prise en considération de la dimension politique de l'action des haut-e-s fonctionnaires. Les recherches de sociologie politique sur la mise en œuvre des lois sur la parité et le travail de l'élu-e, sur la conscience de genre et son incidence sur les pratiques professionnelles, ainsi que sur le genre des politiques publiques, sont riches d'enseignements pour penser le «genre de l'administration».

\footnotetext{
${ }^{1}$ Les auteures participent à un projet, financé par la DGAFP, qui devrait permettre d'apporter des éléments empiriques susceptibles d'étayer ces pistes. Alban Jacquemart et Fanny Le Mancq collaborent également à cette enquête.
} 


\section{La progressive levée des barrières à l'entrée de la fonction publique}

Les avancées des femmes dans l'administration doivent être reliées à l'histoire des droits des femmes dans les sphères économique, scolaire, familiale et politique. L'histoire du travail des femmes sur le long 20e siècle montre que les femmes n'ont pas seulement occupé des postes subalternes dans les administrations ; elles ont eu un accès précoce à des responsabilités mais dans des secteurs et tâches très limités, censés convenir à leur sexe (Schweitzer, 1999). Dès la fin du 19e siècle, des femmes sont présentes dans les fonctions « sociales » de l'Etat : les corps des Inspecteurs de l'enseignement primaire, comme inspectrice des salles d'asile (ancêtres des classes maternelles), ainsi que dans l'Inspection de l'Assistance Publique et du travail. Le corps de l'inspection du travail s'ouvre aux femmes pour la surveillance des ateliers où travaillent des femmes et enfants en 1878 (Clark, 1998). La loi de 1892, qui instaure un concours de recrutement national, écarte toutefois les inspectrices de l'exercice plein du métier, puisqu'elles ne peuvent prétendre au grade d'inspecteur divisionnaire. Dans les années 1920, elles sont toujours exclues des grandes usines comportant des «moteurs complexes ». Il faut attendre le statut de la fonction publique de 1946 pour une égalité statutaire, puis les années 1970 pour l'égalité professionnelle (Schweitzer, 2010). Si la création de l'ENA en 1945 ouvre aux femmes la « grande porte » d'entrée dans la fonction publique, elles sont restées longtemps très minoritaires et ne représentent toujours qu'un tiers des promotions récentes : 26 pour 54 hommes soit $32 \%$ en 2010.

La précocité de l'accès des femmes aux emplois d'encadrement de la fonction publique et la relative ampleur de la féminisation de ces emplois n'implique cependant pas des déroulements de carrière égalitaires. Les mécanismes d'éviction des femmes des hautes sphères sont similaires, voire plus marqués, que ceux observés dans le secteur privé jusqu'à la deuxième moitié du $20^{\mathrm{e}}$ siècle. Alors que des emplois d'encadrement pouvaient être occupés par promotion interne, dès l'entre-deux-guerres, par une minorité de jeunes femmes diplômées d'écoles de commerce (notamment d'HEC Jeunes Filles, créée en 1916), d'ingénieurs (Ecole Polytechnique Féminine créée en 1925), ou d'écoles privées de secrétariat, de comptabilité ou de technicienne de laboratoire, la plupart des emplois de rang A dans la fonction publique leur restaient interdits, de droit ou de fait, dans les ministères les plus féminisés à la base - Culture, Education nationale - comme dans ceux très masculins à tous les niveaux - Industrie, Transports, Intérieur (Rennes, 2007). L'enseignement secondaire et supérieur n'est pas un exemple non plus en matière d'égalité (Cacouault, 2007) : la mixité des agrégations ouvrant sur les carrières du supérieur ne date que de 1974 et la fusion des ENS réservées aux filles - Fontenay et Sèvres - avec celles réservées aux garçons - Saint Cloud et Ulm - est encore plus tardive (1981 et 1986).

La profession policière, encore peu féminisée sur le plan quantitatif (14 \% de femmes en 2005), offre à l'inverse un traitement plus égalitaire des deux sexes, en particulier dans les fonctions supérieures (Pruvost, 2007, 2008). L'entrée des femmes dans les différents grades et corps brise le tabou particulièrement tenace et ancien de l'interdit fait aux femmes d'utiliser des armes. Les premières sont entrées en 1935 à la brigade des mineurs parisienne, munies du diplôme d'assistante sociale, dans une fonction équivalente à celle des inspecteurs. Mais depuis 1968, elles n'y sont plus cantonnées. Contrairement à ce que l'on observe dans l'armée, elles ne sont pas rivées à des tâches administratives 
mais ont accès à toutes les fonctions et tous les grades, à l'exception de ceux de CRS . Paradoxalement, c'est en haut de la hiérarchie qu'elles sont désormais les plus nombreuses (29\% de commissaires en 2005) et les rythmes de progression de leurs carrières suivent étroitement ceux de leurs collègues masculins.

Geneviève Pruvost propose plusieurs pistes d'interprétation de cette situation atypique. La première est celle des imaginaires propres à la profession - contrairement à ce qui est observé ailleurs, l'aptitude des femmes à commander n'est pas mise en cause par les policiers alors que leur insuffisante force physique dans les affrontements directs sur la voie publique est redoutée. La seconde est celle des usages politiques de ces femmes d'exception. Les ministères de l'Intérieur, de droite comme de gauche, mettent en valeur des femmes présentées comme susceptibles de redorer le blason terni de la police depuis les évènements de mai 1968. Les femmes commissaires elles-mêmes tiennent à rester exemplaires tout autant qu'exceptionnelles - elles ne sont pas favorables à une féminisation trop importante et ont tendance à se « viriliser » dans leurs pratiques professionnelles. La troisième piste d'explication des carrières atypiques des femmes commissaires est celle des stratégies matrimoniales d'endogamie et d'hypogamie. La majorité d'entre elles vit en couple avec un fonctionnaire de police. Ce dernier, souvent d'un grade inférieur, soutient leur ambition professionnelle et les suit dans leurs nombreuses mutations géographiques.

\section{Ressources et contraintes des « pionnières » en poste de dirigeantes}

Cette piste de la vie privée et de la «maudite conciliation » entre vie familiale et vie professionnelle ${ }^{2}$ est largement développée par la sociologie du genre pour rendre compte des inégalités sexuées de carrière. Plusieurs recherches se sont intéressées aux propriétés sociales des femmes qui ont accédé à des positions de pouvoir ou à des professions prestigieuses dominées par les hommes, et qui sont donc «atypiques », du point de vue de leur destin de sexe. Les pionnières, telles les premières femmes énarques, sont des héritières plus encore que leurs camarades de promotion. Elles cumulent des héritages culturels et économiques, du côté paternel et maternel (Sineau, 1974). Une enquête explorant la «fabrication familiale de l'excellence », à propos des normaliennes et normaliens scientifiques de la rue d'Ulm, révèle des atouts spécifiques, qui ne se mesurent pas en termes de volume des capitaux mais plutôt en types de configuration familiale d'origine. Education égalitaire des fils et des filles, estime intellectuelle du père et soutien de la mère à une trajectoire d'émancipation professionnelle de la fille (que la mère, diplômée mais au foyer, n'a pu toujours accomplir) éclairent ces trajectoires d'exception (Ferrand, Imbert et Marry, 1999; Marry, 2004). Souvent issues de lignées de « gens du public », et passées par des scolarités brillantes (grandes écoles ou agrégation de droit), les jeunes femmes hauts fonctionnaires seraient cependant moins directement chargées du maintien de la réputation, du nom et du statut social de la famille que leurs frères eux-mêmes engagés dans cette voie d'élite (Bodiguel, 1986).

De nombreuses recherches ont mis en évidence les contraintes spécifiques qui pèsent sur les femmes dans les professions supérieures, salariées ou indépendantes, en raison de l'asymétrie sexuée des charges familiales et de l'alignement de l'organisation du travail

\footnotetext{
${ }^{2}$ Pour reprendre le titre d'un dossier de la revue Travail, genre et sociétés ( $\left.n^{\circ} 24,2010\right)$.
} 
et des modalités de gestion des carrières sur un modèle masculin qui ignore la vie privée. En dépit de leurs diplômes élevés et de leur forte implication dans la vie professionnelle et la carrière, les femmes restent en première ligne dans la responsabilité quotidienne de la famille (conjoint, enfants, parents âgés). L'organisation du travail dans les postes de direction requiert une disponibilité constante, des horaires de travail lourds et souvent imprévisibles, des réunions tardives, des déplacements fréquents en France et parfois à l'étranger, des mobilités géographiques répétées (variable suivant les types d'organisation et de professions) qui rendent difficile la « conciliation » avec une vie de famille. Les moyens financiers et la possibilité de déléguer une partie du travail domestique, variables suivant les professions, ne leur épargnent pas la charge mentale. Les chercheuses en biologie des grands organismes publics (INSERM ou CNRS), qui n'ont pas le même niveau de rémunération que leurs homologues du privé, se stabilisent professionnellement plus tardivement (vers 35 ans en moyenne) et reportent davantage leur maternité pour faire carrière (Marry et Jonas, 2005).

D'autres travaux ont déplacé le regard vers l'avantage que procure aux hommes, le statut de mari et de père. L'ouvrage de François de Singly, Fortune et infortune de la femme mariée (1987) systématise la thèse formulée par Jacqueline Laufer (1982) selon laquelle le rendement du diplôme des hommes et des femmes sur le marché du travail diffère selon leur statut matrimonial : le mariage galvanise les carrières des diplômés masculins, tandis qu'il pénalise celles des femmes. Dans le cas des diplômé-e-s de grandes écoles (Polytechnique, Mines, HEC, ESSEC...), le rôle prédominant du diplôme initial dans les courbes de carrière des grandes entreprises efface pour partie l'effet pénalisant de la vie en couple et des maternités sur les carrières de ces femmes ; mais les hommes mariés et pères sont ceux qui connaissent les promotions les plus rapides aux plus hauts niveaux grâce au soutien d'une épouse diplômée plus disponible et mobile, mais aussi parce qu'ils répondent mieux aux normes attendues de virilité et d'hétérosexualité pour des postes stratégiques de l'organisation (Gadea \& Marry 2000 ; Guillaume \& Pochic 2007).

La sur-représentation des catholiques pratiquants dans les niveaux hiérarchiques élevés de la fonction publique (Darbel et Schnapper, 1969) peut laisser à penser que ces « pères qui gagnent », à la descendance nombreuse, sont pris aussi dans cette logique de la dévotion à la carrière pour maintenir le statut social de leur famille et dans les conditions idéales pour répondre aux attentes de l'organisation (don de soi pour le « bien public », débarrassé de toute contrainte domestique).

Dans certaines entreprises publiques ou secteurs (la banque), la mobilité géographique fréquente et répétée dans des destinations imposées par l'employeur est toujours un comportement attendu des cadres « à potentiel ». Cela tend à favoriser des hommes aux configurations familiales traditionnelles (conjointe ayant abandonné ses ambitions de carrière), certains hommes compensant un diplôme moins prestigieux par ce jeu de la mobilité (Grafmeyer, 1992 ; Bertaux, 2008) et les femmes essayant de résister souvent au prix d'un certain plafonnement (Pochic et Guillaume, 2009b). Si les jeunes cadres ont souvent une vision plus égalitaire des rapports de couple et contestent davantage cette norme de la mobilité géographique qui fragilise leurs aspirations à des carrières en double, ils sont aussi souvent attirés par cette perspective, particulièrement à l'international. Ils/elles voient rarement comment l'entreprise pourrait résoudre cette équation qu'ils/elles estiment personnelle, alors qu'ils sont plus sensibles à la responsabilité de l'employeur sur l'organisation du travail et la « conciliation » avec la vie privée (Guillaume et Pochic 2009). 


\section{Les mécanismes genrés de « façonnage » des élites dirigeantes}

Alors que la sociologie des organisations a longtemps considéré ces dernières comme neutres du point de vue du genre, en raison des normes et procédures bureaucratiques qui sont censées régir leur fonctionnement, des travaux ont au contraire mis en évidence leurs biais de genre (Kanter, 1977 ; Acker, 1992 ; Halford, Savage et Witz, 1997). Dans cette perspective, les déroulements de carrière n'ont plus été expliqués par des contraintes extérieures à la sphère professionnelle (socialisation familiale ou scolaire, rapports de genre au sein du couple), mais ont été réencastrés (embedded) dans des mécanismes genrés de «fabrication » ou « façonnage » des dirigeants par les organisations elles-mêmes, aux différentes étapes de la carrière : recrutement, évaluation du travail et notation de la personne, promotion et affectation. Les biais de genre sont inscrits non seulement dans les procédures formelles de gestion des carrières, apparemment neutres mais en réalité adossés à un référent masculin (critères d'âge ou physiques, disponibilité horaire, mobilité géographique imposée, valorisation différenciée des diplômes, métiers et parcours antérieurs), mais aussi dans les procédures et normes informelles (influence des réseaux de soutien et de cooptation, représentations et stéréotypes associés aux postes de direction variables suivant la « culture » de l'organisation). En ce qui concerne la fonction publique, des enquêtes ont ainsi montré les biais d'un dispositif aussi formellement «méritocratique » et « objectif » que le concours : en amont, qu'il s'agisse de l'administration des impôts (Fortino, 2002) ou des postes de directeurs/directrices d'études à l'EHESS (Backhouche, Godechot et Naudier, 2009), les femmes sont ainsi dissuadées de se présenter et elles sont moins soutenues que les hommes.

A l'instar de ce qui a été analysé dans les entreprises et en politique, les carrières dans la haute fonction publique sont largement façonnées par des procédures informelles, en particulier par les réseaux de cooptation et par des conflits entre filières de métiers plus ou moins prestigieuses qui contrôlent l'accès aux postes de pouvoir. C'est particulièrement le cas des postes des directeurs d'administration centrale, qui sont « à la décision du gouvernement »; même si le recrutement des emplois de directeurs-adjoints, chefs de service et sous-directeurs est moins directement soumis aux changements politiques, il «n'échappe pas pour autant à toute appréciation discrétionnaire du pouvoir politique » (Bodiguel et Quermonne, 1983 : 158). Si les hauts fonctionnaires revendiquent le plus souvent leur neutralité politique (Kessler, 1985), il paraît clair qu'ils doivent leur position non seulement à la reconnaissance de leur « compétence » par l'administration (diplôme prestigieux, appartenance à un grand corps, expérience attendue en terme d'encadrement) mais aussi à leur inscription dans des réseaux politiques, comme l'illustre l'impact des alternance politiques sur le recrutement des hauts fonctionnaires (Lochak, 1986 ; Poirmeur 1986).

Cette nécessité d'articuler carrière professionnelle et affiliation politique, avec le passage valorisé en début de carrière dans les cabinets ministériels (Rouban, 2007) est peut-être une des variables cachées qui permet de mieux comprendre le rétrécissement de la part des femmes constaté par plusieurs études entre les « potentiels promouvables » (cadres A ayant les conditions statutaires et d'ancienneté requises) et les promus dans l'encadrement supérieur, et ce plus particulièrement dans la fonction publique territoriale (Le Pors et Milewski, 2005). Au sein du ministère de l'équipement, pour les postes à responsabilité en DDE, l'administration doit souvent « imposer» une femme contre l'opinion première du préfet et des élus (Doniol-Shaw et Le Douarin, 2003). 
Selon le dernier livre blanc de la fonction publique (Silicani, 2009), l'administration française commence à s'inspirer des politiques de Ressources Humaines du secteur privé, notamment pour l'identification des « hauts potentiels » et la promotion de l'égalité professionnelle. Si les entreprises communiquent beaucoup sur les procédures visant l'égalité qu'elles mettent en place (via des labels et des chartes), les enquêtes des sociologues en relativisent souvent l'impact ou en explicitent les liens avec des préoccupations économiques des employeurs : répondre à une pénurie de main d'œuvre, fidéliser les plus qualifiées, améliorer l'image de l'entreprise auprès des clients (Kirton \& Greene 2000; Laufer 2007). Le caractère rhétorique de nombre de plans d'égalité a été souligné (Rabier 2008), tout comme leur aspect sélectif, aussi bien en terme de profils de femmes que de dimensions de l'égalité concernées. Si les politiques d'égalité peuvent jouer sur les critères de recrutement externe (via des objectifs ou des quotas) et sur la « conciliation » avec la vie familiale (via la prise en charge financière de frais de garde, ou la régulation collective des horaires de réunion), elles peinent à modifier les critères de promotion interne, en terme de compétences requises et de qualités attendues par les cadres opérationnels.

Selon Cynthia Cockburn (1989), qui a comparé les effets de politiques d'égalité des chances mises en œuvre dans les années 1980 en Angleterre dans le secteur privé comme dans le secteur public, ces politiques sont à court terme, formelles et managériales (visant à minimiser les biais de sélection au recrutement et dans la promotion), mais ne visent pas à transformer à plus long terme l'organisation du travail et la distribution du pouvoir ${ }^{3}$. Alison Woodward (1996) montre elle aussi que malgré une promotion formellement « au mérite » et une politique d'égalité des chances offensive depuis 1978, la Commission européenne demeure une organisation masculine-virile. La promotion y dépend de critères informels, dont le soutien d'un parti politique, la nationalité, des loyautés personnelles, et la capacité à se rendre visible. Le harcèlement des stagiaires par des hommes seuls loin de chez eux n'y est pas rare, tout comme le recrutement d'épouses sur statuts précaires, ce qui tend à renforcer les représentations sur le moindre investissement des femmes dans la carrière.

\section{Le New Public Management, opportunité ou risque pour les femmes diplômées ?}

Les réformes qui touchent les modes de gestion du personnel dans la fonction publique, souvent labellisées sous le terme englobant de « Nouveau management public » et cristallisées en France dans la LOLF (Loi Organique relative aux Lois de Finances) puis la démarche de RGPP (Révision Généralisée des Politiques Publiques) initiée en 2007, ont-elles des effets induits favorables ou au contraire négatifs sur les carrières des femmes ? Des travaux menés en Angleterre sur les réformes du service public ou la privatisation des entreprises publiques dès les années 1980, montrent qu'elles ont un effet ambivalent. L'accent mis sur les compétences tertiaires (gestion, finance, GRH, organisation, communication) dans l'évaluation de la performance et les politiques de promotion de l'égalité des chances peut permettre à des femmes diplômées dans ces domaines d'accéder aux premiers niveaux d'encadrement et à des filières de métier émergentes ou revalorisées. Dans la fonction publique hospitalière, une partie des infirmières a

\footnotetext{
${ }^{3}$ Elles peuvent même coexister avec une dégradation des conditions (de salaire, de stabilité et de promotion) pour les femmes en bas de la hiérarchie, en raison de politiques visant la réduction des coûts, comme l'externalisation de certaines activités (réalisées par des sous-traitants ou des contractuels) ou la flexibilisation du travail (temps partiel imposé).
} 
ainsi soutenu les réformes du NHS anglais, puisqu'elles ont entraîné une revalorisation salariale de leurs fonctions après une « job evaluation » (sur le principe du «à travail égal, salaire égal ») et a ouvert pour certain-e-s des possibilités de promotion vers des postes de cadres de direction des hôpitaux (Halford, 2007).

Cependant, ces dynamiques de carrière offertes à certaines femmes ne peuvent se comprendre sans retracer la «structure des opportunités » de promotion et de mobilité dans des organisations en profonde transformation (Halford, Savage \& Witz, 1997) : taille de l'organisation (et postes disponibles, fonction du turn-over et de l'attractivité de l'administration et de la région), cloisonnement des filières professionnelles (et compétition entre segments professionnels pour l'accès aux postes de direction), croissance ou réduction des effectifs (avec des conflits éventuels de « culture » d'organisation en cas de fusion), tensions sur le marché du travail en général (attractivité ou non des postes de fonctionnaires), etc.

La dérégulation et la privatisation du secteur de l'énergie en Angleterre ont ainsi permis l'entrée de femmes dans un secteur traditionnellement masculin et dominé par les ingénieurs, sur des postes de fonctions support (finance, droit, commercial, $\mathrm{RH}$ ) ou pour faire face à des situations périlleuses ou délicates en terme de relations sociales ou de changement organisationnel (parfois pour « désarmer» des syndicats forts). Mais la filialisation et les fusions-restructurations ont encore accentué les difficultés « classiques » pour accéder au dernier cercle de responsabilités, dans un contexte d'intensification du travail, de renforcement des effets de réseaux en contexte de réduction d'effectifs, de fermetures de site avec relocalisations. La légitimité masculine traditionnelle (bureaucratique et technique) s'est trouvée concurrencée par une légitimité plus charismatique et entrepreneuriale, une « masculinité compétitive » loin du discours sur le «management au féminin » (Kerfoot et Knights, 1993 ; Guillaume et Pochic, 2009). Comme l'a souligné Judy Wajcman (1998), le type de management dépend fortement des contextes d'emploi et moins du genre de l'encadrement : on peut être davantage «à l'écoute » dans des organisations en croissance, moins hiérarchisées et laissant place à l'innovation, alors qu'il est plus difficile d'adopter un registre de management « coopératif » dans des contextes de réduction des effectifs et d'éventuel contestations internes, voire de conflit social.

Les réformes actuelles tendent à restructurer fortement les fonctions publiques (en terme de périmètre et d'organisation), et les agents qui y travaillent, en modifiant les qualifications mais aussi les qualités valorisées pour y «faire carrière ». Les effets de la RGPP se font plus particulièrement sentir dans certains ministères, dans les services déconcentrés de l'Etat et en province. La logique des corps entraînait jusqu'alors une séparation et une hiérarchisation très marquée des filières professionnelles, avec une compétition entre corps plus ou moins ouverte, et des corps qui conservaient la main sur un ensemble de « postes réservés » (Thoenig, 1987). Au ministère de l'Equipement, le corps des ingénieurs X-Ponts très masculin avait ainsi des « domaines réservés » (les routes), alors que le corps des ingénieurs des Travaux Publics de l'Etat plus féminisé suivait des carrières moins tracées sur des domaines plus innovants mais moins prestigieux, comme l'urbanisme (Doniol-Shaw et Le Douarin, 2003).

Dans ce contexte, les femmes X-Ponts, très minoritaires, préféraient éviter les situations de « concurrence directe » avec les collègues hommes issus des mêmes filières, et briguaient des postes dans le secteur privé. Les femmes issus des corps minoritaires (ingénieures TPE notamment) ont investi l'espace libre laissé par les X-Ponts dans les services déconcentrés techniques, contribuant à la féminisation des fonctions de directeur régional « comme si 
cela permettait de remplir d'un coup plusieurs cases de quotas implicites » (Jeannot, 2010 : 104) Mais selon cet auteur, la récente réorganisation territoriale des services fusionnés (Equipement, Industrie, Environnement) risque de mettre un terme à cette situation, en ravivant la concurrence entre cadres (avec plus d'un poste sur deux supprimés) et de renforcer les critères d'affiliation politique et de visibilité.

De même, au sein du ministère des Affaires Etrangères et européennes (issus de la fusion entre le ministère des Affaires étrangères et le ministère de la Coopération), la gestion par indicateurs de performance accentue le clivage entre deux filières professionnelles : elle donne plus de légitimité au travail de gestion administrative des cadres consulaires (considéré jusqu'alors comme un travail « ingrat»), alors que les cadres de chancellerie défendent une appréciation plus qualitative de leur travail politique ou culturel, travail « noble» jusqu'alors prisé par les énarques (Boussard et Loriol, 2008). Elle fait aussi émerger une nouvelle spécialité (la gestion), où les cadres spécialisés en logistique du ministère de la coopération peuvent espérer mieux se valoriser.

Enfin, l'accès aux fonctions de direction d'un ministère comme Bercy, où la promotion par voie interne est quasi impossible (Eymeri, 2001) doit se comprendre au regard de la « structure des opportunités » des mobilités entre secteur public et secteur privé. Comme l'a montré Luc Rouban (2010), la pratique du « pantouflage » de l'IGF (Inspection Générale des Finances) prend une forme plus précoce depuis les années 1990, avec un éventuel « rétro-pantouflage » (un retour dans le public). Ces parcours de mobilité sont à relier aux effets d'aubaine dans le privé (libéralisation activités bancaires, fusions-acquisitions et filialisations) et au plafonnement dans la haute fonction publique (vagues de départs en cas d'alternance politique, avec un pic en 2007 en raison d'une plus grande politisation des nominations), parfois lié à l'anticipation de la réduction des postes (comme en 2007 avec la fusion de la Direction Générale des Impôts et de la Direction Générale de la Comptabilité).

Ces changement organisationnels peuvent-ils servir de tremplins pour des femmes (appel d'air en cas de départs des candidats masculins vers le privé, revalorisation de filières professionnelles), ou vont-elles surtout servir « d'alibi » de l'égalité des chances (tout en étant sur-sélectionnées en terme de profil scolaire et social)?

Les études de science politique sur le genre apportent sur la question du genre dans l'encadrement supérieur de la fonction publique des éclairages complémentaires à ceux de la sociologie du travail, à partir d'une prise en considération de la dimension politique de l'action des hauts fonctionnaires. Celle-ci sera envisagée ici sous trois angles : comparaison possible avec le travail d'élu(e), qui a fait l'objet d'amples études dans le contexte de la mise en œuvre des lois sur la parité ; étude de la conscience de genre et de la dimension militante de l'action ; et question de l'impact du genre de l'administration sur le genre des politiques publiques.

\section{Genre, champ politique et bureaucratie}

Les travaux sur le genre dans le champ politique sont des points d'appui indispensables pour comprendre comment joue le genre dans l'encadrement supérieur de la fonction publique, pour deux raisons principales. D'une part, on a déjà évoqué à quel point les carrières dans la très haute fonction publique se caractérisent par l'imbrication étroite de logiques politiques et bureaucratiques. Femmes et hommes de l'élite administrative partagent certains traits avec celles et ceux de l'élite politique (dont le passage par l'ENA est emblématique), circulent d'un espace à l'autre, et il est souvent nécessaire de prendre en compte ces deux mondes 
pour saisir comment se construisent leurs carrières différenciées (Achin et Lévêque, 2007). D'autre part, les travaux menés en science politique depuis le début des années 2000 sur les effets de la loi dite sur la parité en politique (loi du 6 juin 2000) éclairent l'analyse des effets des dispositifs d'égalité mis en place dans la haute fonction publique à peu près au même moment.(Bereni et Revillard, 2007)

Ce sont tout d'abord les travaux sur les carrières des femmes de l'élite politique qui sont susceptibles d'éclairer l'analyse de la construction genrée des carrières dans la haute fonction publique. Les enquêtes de Mariette Sineau, centrées sur les femmes qui ont accédé aux échelons les plus prestigieux de la représentation politique, ont mis au jour les mécanismes qui, nichés dans le fonctionnement des partis, le mode de scrutin, les filières de recrutement des élites politique sous la Cinquième République, (re)produisent l'illégitimité et l'exclusion politique des femmes près de cinquante ans après l'octroi de la citoyenneté politique formelle (Sineau, 1988; Sineau, 2001). L'identité féminine est perçue comme une transgression du rôle politique, dont le caractère andro- et hétérocentré est masqué par la prégnance du discours universaliste. La prégnance d'une culture universaliste de l'intérêt général, le rejet des « différences » et des « particularismes » constituent, sans doute, des caractéristiques historiquement partagées par les univers bureaucratiques et politiques français, susceptibles de jouer comme des contraintes pour les femmes, en invisibilisant les rapports de pouvoir liés au genre et en rendant difficile la mobilisation de l'identité sexuée pour les contester.

Depuis le vote de la loi sur la parité (loi du 6 juin 2000), une nouvelle série de recherches en science politique s'est intéressée aux effets de cette réforme sur les rapports de genre en politique. Lors des débats publics qui ont précédé l'adoption de la réforme, les arguments valorisant la différence des sexes ont prévalu sur les arguments égalitaires (Bereni et Lépinard, 2004 ; Lépinard, 2007). La consécration durable de la rhétorique de la complémentarité des sexes en politique, combinée à la féminisation substantielle d'une partie des assemblées élues (essentiellement au niveau municipal et régional) sous l'effet de la contrainte paritaire, a ouvert des opportunités en même temps qu'elle a porté de nouvelles contraintes pour les femmes dans le champ politique (Achin et al.., 2007). Les qualités traditionnellement associées à l'identité féminine, comme la douceur, la proximité, l'altruisme, ont été portées au devant de la scène, surtout dans les contextes électoraux locaux, où la loi sur la parité s'applique de manière drastique. Mais cette nouvelle « ressource » politique que constitue l'affirmation d'une identité féminine s'est avérée ambivalente, se retransformant en stigmate à mesure que les enjeux politiques s'élèvent et que l'on s'éloigne de l'échelon local, traditionnellement plus ouvert à la « société civile» incarnée par les femmes.

Des recherches émergentes s'intéressent aussi à la manière dont l'identité masculine se trouve reconfigurée dans le contexte paritaire : la « virilité privilège », attributs de l'élite politique traditionnelle, qui n'a guère besoin d'être « ostensiblement et activement travaillée » pour incarner l'universel, s'est trouvée concurrencée, dans le contexte paritaire renforçant la saillance de la différence des sexes, par une forme de «virilité ressource », performée ostensiblement comme une source de bénéfice (Achin et Dorlin, 2007). Ces analyses développées en science politique font échos à celles proposées dès les années 1980 par Jacqueline Laufer (1982) en sociologie du travail, montrant comment les femmes cadres pouvaient « se soumettre », « neutraliser », « valoriser », voire « revendiquer » leur différence, en lien avec le degré de féminisation du secteur dans lesquelles elles évoluaient. Pour comprendre les stratégies différenciées des femmes et hommes cadres 
dans les univers de pouvoir administratif, il nous semble donc essentiel d'étudier leurs rapports complexes aux normes de genre.

Les dispositifs d'égalité mis en place depuis le début des années 2000 dans la haute fonction publique portent la marque de la « grammaire paritaire » issue du champ politique (Bereni \& Revillard 2007), tout comme ils s'inspirent directement de politiques égalité professionnelle relancées dans les entreprises en France depuis $2001^{4}$ et sont influencés par l'Union européenne. Les termes «parité » et "paritaires »s'y sont diffusés comme synonymes d'égalité ; le comptage statistique des hommes et des femmes aux différents échelons de l'administration s'y est imposé comme le principal indicateur de l'inégalité à redresser, et l'objectif numérique de « représentation équilibrée » (voire de «parité »), a été érigé comme l'indicateur par excellence de l'horizon à atteindre en matière d'égalité, le cas échéant au moyen de quotas 5 . Il sera donc intéressant d'analyser, en s'appuyant sur le contrepoint fourni par le champ politique (parallèlement à celui des entreprises), la manière dont la grammaire paritaire a « voyagé » dans la haute administration. L'hypothèse que l'on pose ici est que l'acclimatation de cette grammaire s'opère à l'intersection de deux injonctions contradictoires de la culture bureaucratique : celle de la représentation, partagée avec le champ politique, qui conduit l'administration à chercher à refléter la communauté des citoyens, et notamment, sous l'injonction de la parité, à refléter la différence hommesfemmes ; et celle de l'aveuglement à la différence, qui repose sur des dispositifs de recrutement et de promotion (concours, avancement à l'ancienneté...) refoulant apparemment toutes les caractéristiques « particulières », notamment la différence des sexes.

\section{Conscience de genre et pratiques professionnelles des haut(e)s fonctionnaires}

Une dimension relativement négligée des études sur le «plafond de verre » dans les entreprises est celle de la conscience de genre et/ou féministe (Klein, 1984; Tolleson Rinehart, 1992), d'une part des personnes chargées de la mise en œuvre des politiques d'égalité professionnelle et plus largement des femmes cadres. Dans quelle mesure ces femmes s'identifient-elles subjectivement au groupe des femmes ? Cette conscience de genre s'accompagne-t-elle d'une remise en question de la position subordonnée assignée à ce groupe (conscience féministe) ? Et se traduit-elle en actions, pouvant être assimilées à une forme d'activisme féministe à l'intérieur de l'Etat (Chow, 1987 ; Halford, 1992 ; McBride et Mazur, 2008) ?

Le stigmate associé au terme « féministe » est particulièrement fort dans le contexte politique français, et la volonté de se prémunir vis-à-vis de cette qualification est susceptible d'être d'autant plus marquée dans la haute administration, ce stigmate venant s'ajouter à la « réserve » (vis-à-vis de toute orientation politique ou militante affichée) qu'impose le statut de haut fonctionnaire. Dans l'appréciation de la conscience féministe des haut-e-s fonctionnaire-s, il convient donc d'être attentif aux prises de position féministes susceptibles de s'affirmer dans les entretiens en dépit du refus de l'auto-qualification comme " féministe » (ce que résume le fameux « Je ne suis pas féministe, mais... »). Dénonciation de

\footnotetext{
${ }^{4}$ Avec la loi Génisson du 9 mai 2001, la loi sur l'égalité salariale du 23 mars 2006, la loi Copé-Zimmerman sur les quotas dans les conseils d'administration et de surveillance des entreprises du 27 janvier 2011. ${ }^{5}$ Comme c'est le cas en ce qui concerne les jurys de concours depuis 2008 et sans doute bientôt pour les cadres dirigeants, comme le propose le rapport de Françoise Guégot de mars 2011.
} 
discriminations, d'inégalités, de rapports de pouvoir plus ou moins théorisés (de la reprise éventuelle de théories ou de résultats de sciences sociales à des constats empiriques plus ponctuels tels que le récit d'un refus de promotion qu'on attribue au sexe de l'aspirante, ou encore le sentiment qu'en réunion, la parole des femmes « vaut» moins que celle des hommes) sont autant de signes d'une conscience féministe ${ }^{6}$.

Au-delà des représentations, cette conscience féministe se traduit-elle dans des pratiques ? Le fait que les pratiques professionnelles se trouvent teintées d'un engagement féministe peut avoir deux types d'effets : non seulement une incidence sur le statut des professionnel-le-s eux-mêmes (amélioration de la place des femmes, transformation des relations de travail), mais aussi des effets plus « externes », par lesquels la conscience féministe pourra influer sur l'orientation des politiques publiques dans un sens plus attentif à l'enjeu de l'égalité des sexes (Katzenstein, 1998). Des femmes de l'encadrement de la fonction publique d'Etat américaine ont pu ainsi utiliser leur statut d'insiders pour faire changer un certain nombre de politiques publiques dans un sens plus égalitaire, par exemple en matière d'emploi ou d'éducation (Banaszak 2010). Les travaux sur les instances étatiques dédiées à la promotion des droits des femmes (Mc Bride Stetson et Mazur, 1995; McBride et Mazur 2010) peuvent également être relus sous cet angle, bien qu'ils n'analysent généralement pas les instances concernées comme des instances militantes, mais plutôt comme des « relais » des mobilisations collectives (Banaszak, 2010 ; Bereni et Revillard, 2011). Cette présence féministe dans l'Etat a été analysée comme le prolongement de sociabilités militantes féministes à l'extérieur de l'Etat (Banaszak, 2010) ou comme la manifestation de réseaux et collectifs plus ou moins formels dédiés à la cause des femmes à l'intérieur de l'Etat - des collectifs qui forment, selon la conceptualisation de Laure Bereni, le pôle administratif de «l'espace de la cause des femmes » (Bereni, 2009). Le recensement des pratiques féministes au sein de la haute administration, qui reste à faire ${ }^{7}$, permettra ainsi d'enrichir la compréhension des répertoires d'action féministes.

\section{Du genre de l'administration au genre des politiques publiques}

Volet essentiel des travaux sur le genre en science politique, l'analyse des politiques publiques au prisme du genre a mis en évidence les effets différenciés de ces politiques sur les hommes et les femmes, ainsi que leur rôle dans la constitution, l'entretien ou à l'inverse la subversion des normes et des inégalités de genre (Dandurand, Jenson et Junter, 2002; Mazur, 2002; Jenson et Lépinard, 2009). Si, du fait de leur impact manifeste sur la division sexuée du travail et l'autonomie des femmes, les politiques sociales constituent le principal domaine d'investigation de ces travaux (Morel 2007), d'autres politiques moins évidemment « genrées » ont été analysées sous ce prisme de manière très fructueuse, à l'instar du travail de Marylène Lieber sur les politiques de sécurité (Lieber, 2008). L'idée

\footnotetext{
${ }^{6}$ Tout en notant que les cadres A sont relativement syndiqués par rapport aux cadres du privé, notamment à la CFDT qui a été une organisation syndicale pionnière concernant l'égalité hommes-femmes dans les années 1970, ce qui peut avoir des conséquences sur une éventuelle prise de conscience féministe (via la lecture de la presse syndicale, la formation syndicale, l'exposition à des rassemblements ou manifestations, etc...).

${ }^{7}$ II s'agit en effet de pratiques peu documentées, même si un récent essai de Brigitte Grésy nous fournit un aperçu de ce que de telles pratiques pourraient être Grésy, Brigitte (2009), Petit traité contre le sexisme ordinaire, Paris, Albin Michel.
} 
selon laquelle toutes les politiques publiques peuvent être analysées au prisme du genre a par ailleurs trouvé une consécration institutionnelle dans une évolution de la conception même des politiques d'égalité des sexes avec la démarche de gender mainstreaming, visant une prise en compte des inégalités entre femmes et hommes dans la définition, la mise en œuvre et l'évaluation de toutes les politiques (Jacquot, 2009).

Pour caractériser cette dimension de genre dans les politiques publiques, la démarche la plus fréquente est une démarche de comparaison internationale, conduisant à l'identification de différentes orientations possibles des politiques du point de vue du genre qui font ensuite l'objet de typologies en grands « modèles » ou « régimes » (celles-ci s'inscrivent dans le prolongement critique de la démarche typologique d'Esping-Andersen) (Lewis, 1992; Sainsbury, 1996). L'étude, à un niveau plus microsociologique, des représentations de genre qui ont cours dans la haute administration, est à même de compléter utilement ces travaux comparatifs situés à une échelle très macro, à partir de l'étude d'une catégorie d'acteurs qui contribue à porter, forger et éventuellement transformer les orientations dont les travaux plus macro diagnostiquent l'existence sous une forme plus consolidée. Les cadres dirigeants de la fonction publique constituent en effet des acteurs essentiels non seulement de la mise en œuvre, mais aussi de la définition des politiques publiques (Eymeri, 2003 ; Dreyfus et Eymeri, 2006). On peut donc légitimement se poser la question des effets du genre de l'administration sur le genre des politiques publiques. Dans quelle mesure la masculinité hégémonique (Connel,12005) dans certaines administrations entretient-elle les biais masculins des politiques publiques que ces administrations contribuent à façonner ? A l'inverse, la présence de femmes, et notamment de féministes, dans les hautes sphères de la fonction publique, peut-elle participer d'une remise en question de ces biais ? (Mazur, 2009 ; Banaszak, 2010).

Au-delà de l'hypothèse générale d'un effet de «masse critique » (Kanter, 1977 ; Lovenduski, 2005), ou d'un lien qui pourrait exister entre représentation « descriptive » et « substantive » des femmes dans les politiques publiques (Pitkin, 1967 ; Mcbride Stetson et Mazur, 1995), il s'agit de saisir les mécanismes par lesquels les normes de genre propres à l'organisation et la conscience de genre ou féministe des acteur(rice)s affectent l'orientation de l'action de ces derniers, produisant ainsi des effets de genre sur les politiques publiques. Dans quelle mesure la révolte contre des inégalités professionnelles vécues (conscience féministe) peut-elle servir de moteur à une mobilisation contre ces inégalités (voire contre les inégalités de genre au sens large) y compris au-delà du seul milieu de travail de la personne concernée ${ }^{8}$ ? L'expérience de la subordination pourra aussi avoir l'effet inverse, favorisant une réaction consistant à faire «profil bas » sur les questions de genre, donc à ne pas investir l'enjeu, voire à le faire dans un sens conservateur, en lien avec la nécessité d'établir sa propre légitimité professionnelle dans une organisation fortement genrée. On ne peut donc faire d'hypothèse unilatérale à ce sujet ; tout au plus peut-on faire le pari de l'intérêt qu'il y a à mettre en relation ces deux dimensions (genre de l'administration et genre des politiques publiques) jusqu'ici essentiellement étudiées de façon disjointe.

\footnotetext{
${ }^{8}$ L'expérience personnelle d'inégalités dans la sphère professionnelle (secteur privé ou administration publique) ou dans la sphère partisane-électorale a constitué un moteur non négligeable de l'engagement chez de nombreuses actrices du « féminisme d'Etat " (ministres chargées des femmes, actrices de la haute administration, agentes travaillant pour les structures administratives chargées de la politique à l'égard des femmes), notamment parmi les premières (Revillard, 2007).
} 


\section{Conclusion}

Au terme de cette revue de la littérature sur le «plafond de verre », il nous semble évident que l'analyse des inégalités de genre dans l'accès aux postes les plus élevés de la fonction publique doit bénéficier du croisement de perspectives de recherche qui, jusqu'à aujourd'hui, ont trop peu dialogué entre elles : la sociologie du travail et des organisations d'une part, et l'analyse des rapports entre genre, politique et action publique d'autre part. La combinaison de ces deux perspectives permet en effet de saisir ce qui, dans les dynamiques de genre dans la haute fonction publique, relève de mécanismes constatés dans d'autres milieux de travail à dominante masculine, tout en prenant en considération les spécificités liées à la dimension politique de l'activité concernée. Le questionnement relatif à la conscience de genre/conscience féministe des femmes dans la haute fonction publique, et aux effets de cette orientation sur leur activité, est par ailleurs à même d'enrichir l'analyse de l'évolution des rapports de genre y compris dans des sphères professionnelle à teneur moins politique. L'étude empirique des inégalités de genre dans la haute fonction publique revêt ainsi, en retour, un caractère heuristique tant pour la sociologie du travail et des organisations que pour la science politique.

\section{Bibliographie}

ACHIN C. et DORLIN E., (2007 ), « J'ai changé, toi non plus ». La fabrique d'un-e présidentiable : Sarkozy/ Royal au prisme du genre », Mouvements, http://www.mouvements.asso.fr.

ACHIN C. et LEVEQUE S., (2007), Femmes, énarques et professionnelles de la politique. Des carrières exceptionnelles sous contrainte, Genèses 67, 24-44.

ACKER J., (1992), Gendering Organizational Theory in Mills A. et Tancred P. (eds.), Gendering Organizational Analysis, Sage Publications, Newbury Park, 248-260.

BACKOUCHE I., GODECHOT O. et NAUDIER D., (2009), Un plafond à caissons : Les femmes à 1'EHESS, Sociologie du travail, 51 (2), 253-274.

BANASZAK L. A., (2010), The women's movement inside and outside the state, Cambridge University Press Cambridge.

BERENI L., (2009), Quand la mise à l'agenda ravive les mobilisations féministes. L'espace de la cause des femmes et la parité politique (1997-2000), Revue française de science politique, 59 (2), 301-323.

BERENI L. et REVILLARD A., (2007), Des quotas à la parité : féminisme d'Etat et représentation politique (1974-2007), Genèses 67, 5-23.

BERENI L. et REVILLARD A., (2011), Contentious institutions: rethinking the movement/state intersection, Second European Conference on Politics and Gender, Central European University, Budapest (13-15 janvier).

BERTAUX-WIAME I., (2006), Conjugalité et mobilité professionnelle : le dilemme de l'égalité, Cahiers du genre 41, 49-73.

BODIGUEL J.-L., (1986), La socialisation des hauts fonctionnaires. Les directeurs d'administration centrale, in D. Lochak et al. (dir.), Haute administration et politique, PUF, Paris, 81-99.

BODIGUEL J.-L. et QUERMONNE J.-L., (1983), La haute fonction publique sous la Ve République, PUF, Paris.

BOUSSARD V. et LORIOL M., (2008), Les cadres du Ministère des affaires étrangères et européennes face à la LOLF, Revue Française d'Administration Publique 128, 717-728.

BUSCATTO M. et MARRY C.,(2009), Le plafond de verre dans tous ses éclats : la féminisation des professions supérieures au XX' ${ }^{\mathrm{e}}$ siècle, Sociologie du travail 51 (2), 170-182. 
CACOUAULT-BITAUD M., (2007), Professeurs... mais femmes - Carrières et vies privées des enseignantes du secondaire au $X X^{\mathrm{e}}$ siècle, La Découverte Paris.

CLARK L. L., (1998) Les carrières des inspectrices du travail, 1892-1939 in Robert J.-L., Inspecteurs et Inspection du travail sous la III et la IV République, La Documentation française, Paris, 128-135.

COCKBURN C., (1989), Equal opportunity : the short and the long agenda, Industrial Relations Journal 20 (3), 213-225.

CONNELL R. W., (2005), Masculinities, University of California Press, Berkeley.

DANDURAND R. B., JENSON J.. et JUNTER A., (2002), Présentation : Les politiques publiques ont-elles un genre?, Lien Social et Politiques (47), 5-13.

DARBEL A., SCHNAPPER D., (1969), Les agents du système adminstratif, Mouton, Paris.

DONIOL-SHAW G. et LE DOUARIN L., (2003), Les freins à l'accès des femmes aux postes d'encadrement supérieur du Ministère de l'Equipement, des Transports, du Logement, du Tourisme et de la Mer, Rapport de recherche, LATTS, Marne-la-Vallée.

DREYFUS F. et EYMERI J.-M. (dir.), (2006), Science politique de l'administration. Une approche comparative, Economica, Paris.

EYMERI J.-M., (2003), Frontière ou marches? De la contribution de la haute administration à la production du politique, in Lagroye J. (ed.), La politisation, Belin/, Paris, 47-77.

FERRAND M., IMBERT F. et MARRY C., (1999), L'excellence scolaire : une affaire de famille, Le cas des normaliennes et normaliens scientifiques, L'Harmattan, Paris.

FORTINO S. (2002), La mixité au travail, La Dispute, Paris.

GADEA C. et MARRY C., (2000), Les pères qui gagnent : descendance et réussite professionnelle chez les ingénieurs, Travail, Genre et Sociétés 3, 109-135.

GRAFMEYER Y., (1992), Les gens de la banque, PUF, Paris.

GUILLAUME C. et POCHIC S., (2007), La fabrication organisationnelle des dirigeants. Un regard sur le plafond de verre, Travail, Genre et Sociétés 17, 79-103.

GUILLAUME C. et POCHIC S., (2009), Des discours sur la conciliation en tension avec les normes de carrière. L'exemple des cadres d'entreprise, in Nicole-Drancourt C. (ed.), Conciliation travail - famille : attention travaux, L'Harmattan, Paris 113-125

HALFORD S., SAVAGE M. et WITZ A., (1997), Gender, careers and organisations. Current developments in banking, nursing and local government, Macmillan, London.

HALFORD S., (1992), Feminist change in a patriarchal organisation : the experience of women's initiatives in local government and implications for feminist perspectives on state institutions, in Savage M., Witz A. (ed.), Gender and bureaucracy, Blackwell, London, 155-185.

HALFORD S., (2007), Changement organisationnel et stratégies identitaires. Le cas de cinq infirmières britanniques, Travail, Genre et Sociétés 7, 27-48.

JACQUOT S., (2009), La fin d'une politique d'exception. L'émergence du gender mainstreaming et la normalisation de la politique communautaire d'égalité entre les femmes et les hommes, Revue française de science politique 59 (2), 247-278.

JEANNOT G., (2010), Sélection et parcours des directeurs régionaux des services déconcentrés techniques, Sociologies pratiques 210, 97-110.

JENSON J. et LEPINARD E., (2009), Penser le genre en science politique. Vers une typologie des usages du concept, Revue française de science politique 59 (2), 183-202.

KANTER R. M. (1977), Men and Women of the Corporation, Basic Books, New York.

KATZENSTEIN M. F., (1998), Faithul and Fearless. Moving from Feminist Protest Inside the Church and the Military, Princeton University Press, Princeton.

KERFOOT D., KNIGHTS D., (1993), Management, masculinity and manipulation: from paternalism to corporate strategy in Financial services in Britain, Journal of Management Studies 30 (4), 659-678. 
KESSLER M.-C., (985), La psychologie des hauts fonctionnaires, in CURAPP, Psychologie et science administrative, PUF, Paris, 157-175.

KIRTON G. et GREENE A.-M., (2000), The Dynamics of Managing Diversity, a critical approach, ButterworthHeinemann, Oxford,

KLEIN E., (1984), Gender politics. From consciousness to mass politics, Harvard University Press, Cambridge.

LAUFER J., (1982), La féminité neutralisée ? Les femmes cadres dans l'entreprise, Flammarion, Paris.

LAUFER J., (2004), Femmes et carrières : la question du plafond de verre, Revue Française de Gestion 151, 117-128.

LAUFER J., (2007), L'égalité professionnelle hommes femmes: une mise en perspective, in F. Barth et C. Falcoz (dir.), Le management de la diversité. Enjeux, fondements et pratiques, L'Harmattan, Paris, 25-49.

LEWIS J., (1992), Gender and the development of Welfare regimes, Journal of European Social Policy 2 (3), 159-173.

LIEBER M., (2008), Genre, violences et espaces publics. La vulnérabilité des femmes en question, Presses de Sciences Po, Paris.

LOVENDUSKI J., (2005), Feminizing politics, Polity Press, Cambridge.

MARRY C., (2004), Une révolution respectueuse : les femmes ingénieurs, Belin, Paris, 287p.

MARRY C. et JONAS I., (2005), Chercheuses entre deux passions : l'exemple des biologistes, Travail, genre et sociétés 14, 69-88.

MARRY C. et LE MANCQ F., (2011, à paraître), La dynamique inachevée de l'égalité entre les sexes, in Galland O. et Lemel Y. (dir.), Société Française, Pesanteurs et mutations, Armand Colin, Paris.

MAZUR AMY G., (2002), Theorizing feminist policy, Oxford University Press, Oxford.

MCBRIDE D. et MAZUR, A. G. (eds.); (1995), Comparative state feminism, Sage, Thousand Oaks.

MCBRIDE D. et MAZUR, A. G. (eds.), (2010), The politics of state feminism. Innovation in comparative research, Temple University Press, Philadelphia.

MOREL N., (2007), Le genre des politiques sociales. L'apport théorique des 'gender studies' à l'analyse des politiques sociales, Sociologie du travail 49 (3), 383-397.

PITKIN H. F., (1967), The concept of representation, University of California Press, Berkeley.

POCHIC S. et GUILLAUME C., (2009a), Les carrières des cadres au cœur des restructurations: l'ambivalence des effets de genre, Sociologie du travail 51 (2), 275-299.

POCHIC S. et GUILLAUME C., (2009b), Les attendus implicites de la carrière : usages et mises en forme de la vie privée, in Berrebi-Hoffmann Isabelle (ed.), Politiques de l'intime - des utopies d'hier aux mondes du travail d'aujourd'hui, La Découverte, Paris, 145-167

PRUVOST G., (2007), La dynamique des professions à l'épreuve de la féminisation : l'ascension atypique des femmes commissaires, Sociologie du travail 49 (1), 84-99.

PRUVOST G., (2008), De la sergote à la femme flic, une autre histoire de l'institution policière, La Découverte, Paris.

RABIER M., (2009), La négociation sur l'égalité professionnelle entre les femmes et les hommes, in DARES/ DGT, Le bilan de la négociation, p. 313-330

RENNES J., (2007), Le mérite et la nature. Une controverse républicaine : l'accès des femmes aux professions de prestige (1880-1940), Fayard, Paris.

REVILLARD A., (2007). La cause des femmes dans l'Etat : une comparaison France-Québec (1965-2007), Thèse de doctorat en sociologie, Ecole normale supérieure de Cachan.

ROUBAN L., (2010), L’inspection générale des finances 1958-2008 : pantouflage et renouveau des stratégies élitaires, Sociologies pratiques (21), 21-34.

SAINSBURY D., (1996), Gender, equality, and welfare states, Cambridge University Press, Cambridge.

SCHWEITZER S., (1999), Quand les femmes représentent l'Etat, Travail, Genre et Sociétés (2), 139-152. 
SCHWEITZER S., (2010), Femmes de pouvoir, une histoire de l'égalité professionnelle en Europe (19-20 siècle), Payot, Paris.

SINEAU M., (1974), Les femmes et l'ENA, Annuaire International de la Fonction Publique 4, 63-110.

SINEAU M., (1988), Des femmes en politique, Economica, Paris.

SINEAU M., (2001), Profession femme politique. Sexe et pouvoir sous la Cinquième République, Presses de Sciences po Paris.

THUILLER P., (1988), Les femmes dans l'administration depuis 1900, PUF, Paris.

TOLLESON RINEHART S., (1992), Gender consciousness and politics, Routledge, New York.

WAJCMAN J., (1998), Managing like a man : Women and men in corporate management, Polity Press, London.

WOODWARD A., (1996), Multinational masculinities and european bureaucracies, in Collinson D. et Hearn J. (eds.), Men as managers - Managers as men. Critical perspectives on men, masculinities and managements, Sage, London. 


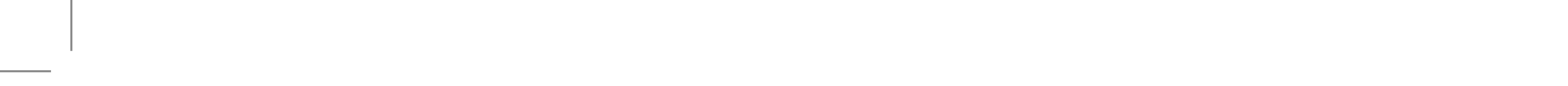

